\title{
Genomic analysis and temperature-dependent transcriptome profiles of the rhizosphere originating strain Pseudomonas aeruginosa M18
}

\author{
Da-Qiang Wu, Jing Ye, Hong-Yu Ou, Xue Wei, Xianqing Huang, Ya-Wen He* and Yuquan Xu*
}

\begin{abstract}
Background: Our previously published reports have described an effective biocontrol agent named Pseudomonas sp. M18 as its $16 \mathrm{~S}$ rDNA sequence and several regulator genes share homologous sequences with those of $P$. aeruginosa, but there are several unusual phenotypic features. This study aims to explore its strain specific genomic features and gene expression patterns at different temperatures.

Results: The complete M18 genome is composed of a single chromosome of 6,327,754 base pairs containing 5684 open reading frames. Seven genomic islands, including two novel prophages and five specific non-phage islands were identified besides the conserved $P$. aeruginosa core genome. Each prophage contains a putative chitinase coding gene, and the prophage II contains a capB gene encoding a putative cold stress protein. The non-phage genomic islands contain genes responsible for pyoluteorin biosynthesis, environmental substance degradation and type I and III restriction-modification systems. Compared with other P. aeruginosa strains, the fewest number (3) of insertion sequences and the most number (3) of clustered regularly interspaced short palindromic repeats in M18 genome may contribute to the relative genome stability. Although the M18 genome is most closely related to that of $P$. aeruginosa strain LESB58, the strain M18 is more susceptible to several antimicrobial agents and easier to be erased in a mouse acute lung infection model than the strain LESB58. The whole M18 transcriptomic analysis indicated that $10.6 \%$ of the expressed genes are temperature-dependent, with 22 genes up-regulated at $28^{\circ} \mathrm{C}$ in three non-phage genomic islands and one prophage but none at $37^{\circ} \mathrm{C}$.

Conclusions: The $P$. aeruginosa strain M18 has evolved its specific genomic structures and temperature dependent expression patterns to meet the requirement of its fitness and competitiveness under selective pressures imposed on the strain in rhizosphere niche.
\end{abstract}

\section{Background}

Previously published work from our group has described an effective biocontrol strain M18 that was isolated from sweet melon rhizosphere in Shanghai suburb in 1996 [1]. This strain has been named as the Pseudomonas sp. M18, as its 16S ribosomal DNA sequence shares a high similarity to that of $P$. aeruginosa [1], as well as high sequence identities of several global regulatory genes, including gacA [2], rpoS [3], qscR [4], rhlI [5], lasI [6] and $\operatorname{rsm} A$ [7]. However, the strain M18 has developed several unusual features which distinguish it from that

\footnotetext{
* Correspondence: yawenhe@sjtu.edu.cn; xuyq@sjtu.edu.cn State Key Laboratory of Microbial Metabolism, School of Life Sciences and Biotechnology, Shanghai Jiao Tong University, 800 Dongchuan Road, Shanghai 200240, PR China
}

(c) 2011 Wu et al; licensee BioMed Central Ltd. This is an Open Access article distributed under the terms of the Creative Commons Attribution License (http://creativecommons.org/licenses/by/2.0), which permits unrestricted use, distribution, and reproduction in any medium, provided the original work is properly cited. of the clinically significant $P$. aeruginosa strains, and indeed are more similar to soil-inhabiting biocontrol Pseudomonas strains, such as P. fluorescence 2-79 [8], Pf-5 [9] and P. chlororaphis PCL1391 [10]. Firstly, the predominant phenazine produced by the strain M18 is phenazine-1-carboxylic acid (PCA) rather than pyocyanin (PYO) [11]. PCA is considered to be a major biocontrol compound [12], while PYO is not necessary for fungal killing [13]. It was determined that more PCA could be produced in the strain $\mathrm{M} 18$ at $28^{\circ} \mathrm{C}$ rather than $37^{\circ} \mathrm{C}$ compared with that produced by the $P$. aeruginosa strain PAO1 [11]. Secondly, the strain M18 is the first strain that was reported to produce two antibiotics, PCA and pyoluteorin (Plt) in one single strain [1]. The production of the two antibiotics of the strain M18 can 
result in synergistic antifungal activities against several phytopathogens. The biosynthesis gene clusters of two antibiotics share 99\% nucleotide sequence identity with the clusters of Liverpool Epidemic Strain P. aeruginosa LESB58. However, a 5-base-pair deletion exists in the pltB gene of Plt synthetic cluster resulting in a frameshift mutation in the strain LESB58 [14]. Thirdly, several interesting features with regards to the regulatory mechanism of the production of these two antibiotics were found to be quite different from that in $P$. aeruginosa. For example, PCA production is negatively regulated and Plt production is positively regulated by a global regulator named GacA [2]. However, the converse relationship occurs in the strain M18 through another global regulator, RsmA [7]. In a more recent study, we demonstrated the negative effect of the QscR regulator on PCA production, but not on Plt in the strain M18 [4]. Studies have also been conducted on the differential regulation of PCA and Plt production by potential quorum sensing (QS) signaling molecule $N$-acyl homoserine lactones (AHLs) secreted by this strain [5,6]. The interrelationship between the QS las and rhl system in strain M18 are also different from that in strain P. aeruginosa PAO1 [15].

Besides the biocontrol strain M18, several $P$. aeruginosa strains, i.e., 7NSK2 [16], PNA1 [17], NJ-15 [18], PUPa3 [19], which inhabit the rhizosphere niche, have been found to show excellent plant growth promoting feature and effective biocontrol property against various phytopathogens, and are utilized for biopesticide development. The genetically modified strain M18 has been recently developed as a high yield antifungal antibiotic PCA producer [20-22]. However, little information is available on their specific genome structures, transcriptome profiles and virulence activities compared to their clinical counterparts.

A series of complete genomic sequences of $P$. aeruginosa strains that originated from patients [14,23-25] have been published recently, and normal environmental $P$. aeruginosa isolates were chosen to compare their virulence and genomic structures [26,27]. To date, none of $P$. aeruginosa strains that originate from the rhizosphere has been completely sequenced and analyzed in detail. This study aims to delineate the genomic structural differences among various $P$. aeruginosa strains that originate from nosocomial patients and rhizosphere niches, and to improve our general understanding of what kinds of strategies have been adopted by the $P$. aeruginosa genome in response to various environment conditions, especially to different temperatures. In this report, the complete genome of the strain M18 was sequenced and compared with those of previously sequenced nosocomial strains. Furthermore, the antimicrobial agent resistance in vitro and the competitive growth ability in a mouse model of acute lung infection in vivo were investigated in the two strains of M18 and LESB58. The temperature dependent genome expression pattern based on the M18 genome was finally analyzed at $28^{\circ} \mathrm{C}$ and $37^{\circ} \mathrm{C}$.

\section{Results and discussion}

\section{Strain M18 is an effective biological control agent}

The strain M18 was isolated from the sweet melon rhizosphere that grew in Shanghai suburb in 1996, and proved to have effective biocontrol activity to protect plants against various phytopathogens infection [1]. In this report, we first provide the evidence to show that the secondary metabolites produced from the strain M18 have strong effective inhibitory activity against the growth of the Mycosphaerella melonis mycelium plug on a potato dextrose agar (PDA) plate (Figure 1A) and therapeutic anti-fungal effect on the sweat melon plant with full recovery of the plant infected by the $\mathrm{M}$. melonis fungus two days after the culture of $10^{8} \mathrm{CFU} / \mathrm{ml}$ of the strain M18 that was sprayed on the infected sweat melon stem (Figure $1 \mathrm{~B}$ and $1 \mathrm{C}$ ). We have already found that the strain M18 has a broad spectrum of anti-fungal activities against various phytopathogens, including $R h i$ zoctonia solani, Sclerotina sclerotiorum, Fusarium oxysporum, Colletotrichum gossypii, Mycosphaerella melonis, Phytophthora capsici, Ralstonia solanacearum and others. The strain M18 also shows effective inhibiting activity against both the Gram-negative bacterium Xanthomonas oryzae pv. Oryzae, and the Gram-positive bacterium Bacillus cereus (data not shown). Therefore, the unusual features of the strain M18 against various fungal phytopathogens and bacteria prompted us to sequence the complete genome of this strain to ascertain more about the genomic structures, with specific reference to this expression pattern at different temperatures through analysis of microarray-based transcriptome profiles.

\section{General genomic features and comparative analysis}

The complete genome of the strain M18 was sequenced and assembled as shown in Figure 2. A general comparison of the genomic features of the strain M18 and other sequenced nosocomial P. aeruginosa strains is shown in Table 1 . The average GC content of the M18 genome is $66.5 \%$, which is remarkably similar to the previously sequenced $P$. aeruginosa strains PAO1, PA14, LESB58 and PA7. The genome of the strain M18 is made of a single chromosome of $6,327,754 \mathrm{bp}$ in size, which is slightly larger than the $6,264,404$ bp of strain PAO1 [23], but relatively smaller than the $6,537,648$ bp of strain PA14 [24], 6,601,757 bp of LESB58 [14] and 6,588,339 bp of strain PA7 [25]. The M18 genome was annotated to contain 5,684 

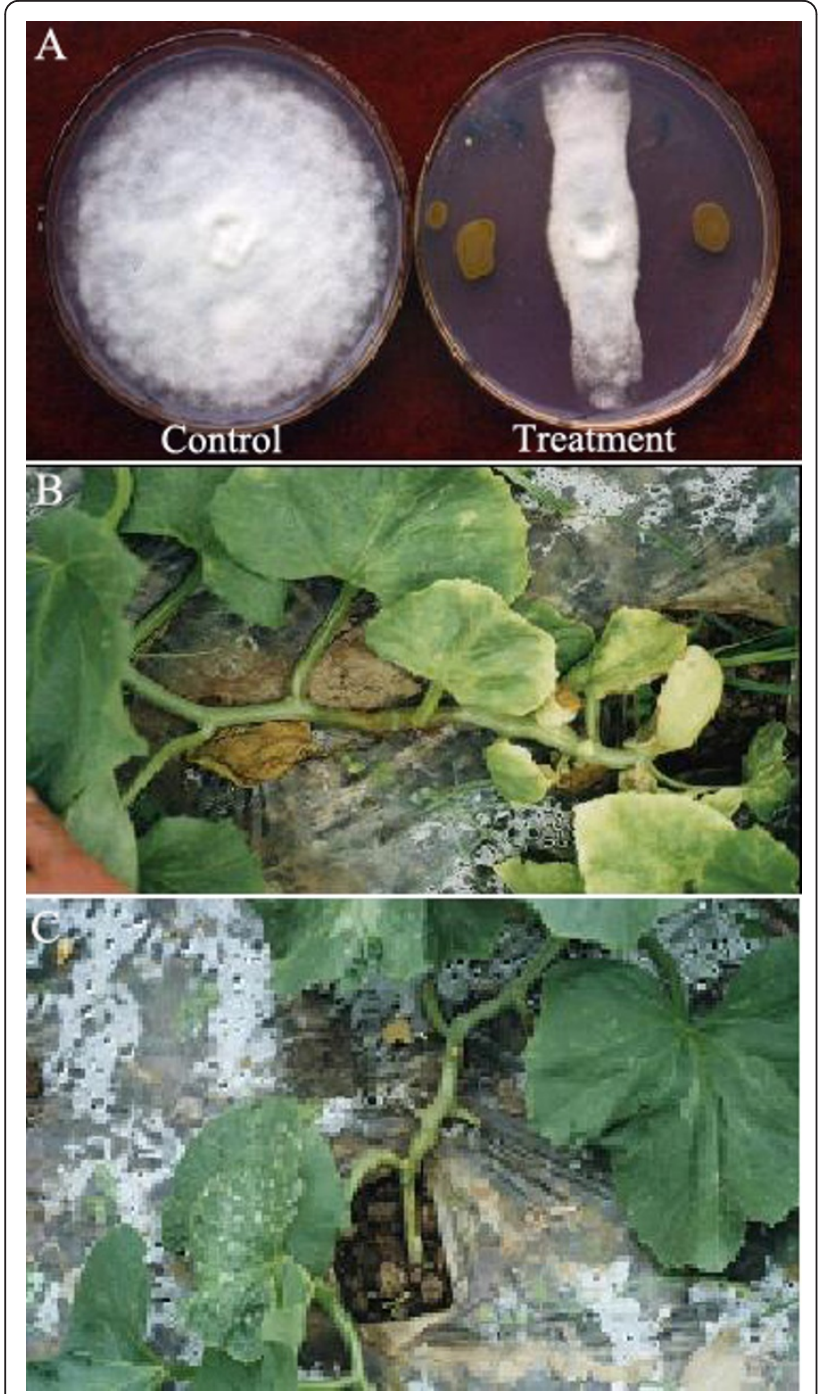

Figure 1 Biological control ability of $P$. aeruginosa M18. The growth of the Mycosphaerella melonis mycelium plug (inoculated in the center of the PDA plate) was completely inhibited by the metabolites produced by $P$. aeruginosa M18 (A). The initial symptom of fungal disease that appeared on the melon plant (B) and fully recovered after it was sprayed with a suspension of the strain M18 culture (C).

open reading frames (ORFs) and 80 RNA genes, which represent $89 \%$ of the total genomic DNA. Using the mGenomeSubtractor online software [28], we found that the genome similarity among these five $P$. aeruginosa strains is very high; however 93 ORFs in the M18 genome, 109 ORFs in PAO1, 256 ORFs in PA14, 380 ORFs in LESB58 and 929 ORFs in PA7 are strain-specific under the threshold of 0.6 of identity or 0.7 of match length/query length. The strain specific genes contained in the M18 genome are listed in Additional file 1 . These results indicated that the available genomes of $P$. aeruginosa strains originating from both clinical and environmental sources contain a highly conserved core genome in large than $90 \%$ of ORFs, with several accessory regions in less than $10 \%$ of ORFs remarkably varied depending on their different environmental niches (except taxonomic outlier PA7).

Global alignments were performed by the MUMer software [29], and the results showed that the large segment inversion of the chromosome by recombination between the rrnA and $r r n B$ genes was found in the M18 genome, as well as in other $P$. aeruginosa strains LESB58, PA14 and PA7 (Figure 3B) in comparison with that in PAO1 genome sequenced by the Seattle consortium [23]. Interestingly, the inversion also exists in different PAO1 sublines which are studied and stored in German and American laboratories, and the phenotypes among the PAO1 sublines are also different [30]. This suggests that some physiological consequences occur among the PAO1 sublines and other $P$. aeruginosa strains because of the inversion [27]

The comparison of the M18 genome and the other available $P$. aeruginosa genomes with regards to the functional category breakdown of coding sequences (CDSs) [31] shows a similar distribution in most groups, but the two strains M18 and LESB58 show a greater degree of similarity than others, including a similar number of genes related to DNA replication, recombination and repair, the transport of inorganic ions and amino acids, amino acid metabolism, and the biosynthesis, transport and catabolism of secondary metabolites (Table 1). This suggests that the two strains share a common ancestor as they have the closest relationship in comparison with all other sequenced $P$. aeruginosa strains. The phylogenetic tree was further generated by maximum parsimony (MP) method, using 58 phylogenetic useful genes in $P$. aeruginosa [27]. The result confirmed that the strain M18 is the closest relative to strain LESB58 among the sequence available $P$. aeruginosa strains, as shown in Figure 3A. We also found that nine out of eleven $\mathrm{O}$-antigen loci in the strain M18 share more than $95 \%$ similarity to the serotype O6 loci in LESB58 [32], indicating that M18 may carry a similar serotype $\mathrm{O} 6 \mathrm{O}$-antigen. Based on these sequence data, the strain M18 is now designated as $P$. aeruginosa M18, a new isolate of $P$. aeruginosa.

\section{Genomic islands and prophages}

In comparison with other sequenced nosocomial $P$. aeruginosa genomes, the unique regions of genomic plasticity were predicted in the M18 genome, including five specific genomic islands (GIs) designated MGI-I to V and two novel prophages, I and II. The general features of these GIs and prophages in the strain M18 are summarized in Figure 4A and Additional file 2. However, we have not found homologies of the pathogenic islands 
$\mathrm{Ob}$

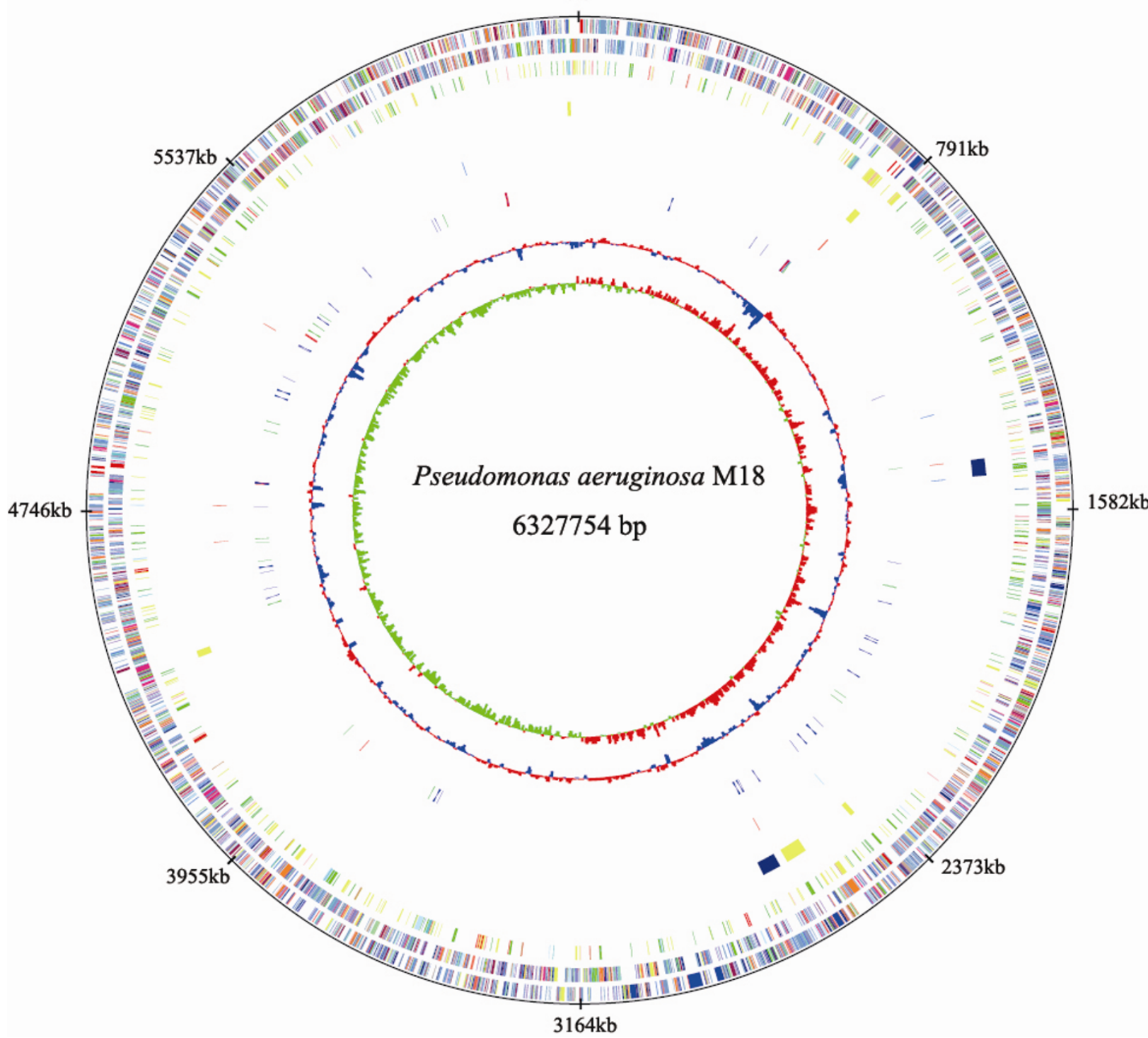

Figure 2 Circular representation of the $P$. aeruginosa M18 genome. The chromosome representation was constructed by GenomeViz [75]. Starting from the outermost circle going inwards: the outermost circles indicate coding sequence (CDS) in plus (circle 1) and minus (circle 2) strands are colored by functional categories, as follows: energy production and conversion (orange); cell division and chromosome partitioning (dark orange); amino acid transport and metabolism (maroon); nucleotide transport and metabolism (antique white); carbohydrate transport and metabolism (yellow); coenzyme metabolism (pink); lipid metabolism (tomato); translation, ribosomal structure and biogenesis (peach); transcription (medium purple); DNA replication, recombination and repair (red); cell envelope biogenesis and outer membrane (green); cell motility and secretion (deep pink); posttranslational modification, protein turnover and chaperones (pale green); inorganic ion transport and metabolism (royal blue); secondary metabolites biosynthesis, transport and catabolism (blue); general function prediction only (dodger blue); function unknown (sky blue); signal transduction mechanisms (cyan); intracellular trafficking and secretion (light blue); defense mechanisms (medium purple). Circle 3 indicates temperature-dependent CDSs colored by fold change of transcriptional level, as follows: up-regulated by more than 5-fold (blue); between 5- and 4-fold (light blue); between 4- and 3-fold (green); between 3- and 2-fold (cyan) at $28^{\circ} \mathrm{C}$ compared with that at $37^{\circ} \mathrm{C}$; transcriptional level down-regulated more than 5-fold (red); between 5- and 4-fold (orange); between 4- and 3-fold (pink); between 3- and 2 -fold (yellow) at $28^{\circ} \mathrm{C}$ compared with that at $37^{\circ} \mathrm{C}$. Circle 4 indicates genomic islands in M18, colored by prophage (blue) and genomic islands (yellow). Circle 5 represents integrase genes (tomato), transposase genes (light blue) and DNA recombinase genes (dodger blue). Circle 6 represents RNA genes colored by tRNA (blue), rRNA (red), and small RNA (green). Circles 7 and circle 8 represent the GC content and skew, respectively. 
Table 1 General genomic features of P.aeruginosa M18 and other P. aeruginosa strains

\begin{tabular}{|c|c|c|c|c|c|}
\hline & M18 & PAO1 & PA14 & LESB58 & PA7 \\
\hline Genome size (bp) & $6,327,754$ & $6,264,404$ & $6,537,648$ & $6,601,757$ & $6,588,339$ \\
\hline GC content (\%) & 66.5 & 66.6 & 66.3 & 66.3 & 66.5 \\
\hline Protein coding genes & 5,684 & 5,566 & 5,892 & 5,925 & 6,286 \\
\hline Protein coding density (\%) & 89 & 89 & 89 & 88 & 89 \\
\hline RNA genes & 80 & 96 & 72 & 102 & 75 \\
\hline Strain-specific genes $(C D S)^{a}$ & 93 & 109 & 256 & 380 & 929 \\
\hline IS & 3 & 12 & 10 & 8 & 15 \\
\hline \multicolumn{6}{|l|}{ CDS assigned function ${ }^{\mathrm{b}}$} \\
\hline Translation, ribosomal structure and biogenesis & 183 & 205 & 205 & 199 & 206 \\
\hline Transcription & 414 & 516 & 537 & 501 & 530 \\
\hline DNA replication, recombination and repair & 127 & 160 & 185 & 145 & 235 \\
\hline Cell division and chromosome partitioning & 35 & 34 & 35 & 34 & 37 \\
\hline Post-translational modification, protein turnover, chaperones & 186 & 200 & 210 & 201 & 215 \\
\hline Cell envelope biogenesis, outer membrane & 242 & 265 & 266 & 261 & 260 \\
\hline Cell motility and secretion & 150 & 150 & 154 & 149 & 152 \\
\hline Inorganic ion transport and metabolism & 294 & 376 & 377 & 313 & 355 \\
\hline Signal transduction mechanisms & 233 & 337 & 345 & 337 & 346 \\
\hline Energy production and conversion & 304 & 329 & 340 & 330 & 336 \\
\hline Carbohydrate transport and metabolism & 196 & 252 & 249 & 196 & 250 \\
\hline Amino acid transport and metabolism & 474 & 587 & 590 & 490 & 571 \\
\hline Nucleotide transport and metabolism & 107 & 108 & 110 & 104 & 105 \\
\hline Coenzyme metabolism & 178 & 191 & 192 & 210 & 192 \\
\hline Lipid metabolism & 202 & 244 & 248 & 234 & 245 \\
\hline Secondary metabolites biosynthesis, transport and catabolism & 140 & 205 & 212 & 171 & 198 \\
\hline Function unknown & 1,578 & 1,465 & 1,706 & 1,768 & 2,139 \\
\hline
\end{tabular}

${ }^{a}$ The strain-specific coding sequences (CDS) were predicted by proteins with low similarity to the other four sequenced $P$. aeruginosa strain genomes (under the expected threshold of 0.6 for identity or 0.7 for match length/query length) using the mGenomeSubtractor [28].

${ }^{\mathrm{b}}$ CDS assigned function was based on the COGs according to RPS-BLAST [66].

against mammals such as PAPI-1 [33], PAPI-2 [34], PAGI-5 [35], LESGI-5 [14], in the M18 genome.

To the best of our knowledge, the two GIs, MGI-I and MGI-II are initially found in $P$. aeruginosa M18, and share 38\% identity with that in Pseudomonas putida GB-1 and 55\% with Nitrosomonas europaea ATCC 19718, respectively. The GC content of MGI-I (51.7\%) and MGI-II (55\%) are quite lower than the average GC content $(66.5 \%)$ of the M18 genome. Difference in the percentage of GC content between the GIs and the core genome is considered as an important marker of horizontal gene transfer, suggesting that these two GIs are associated with recently acquired genetic material. MGII contains two genes (PAM18_0699 and PAM18_0700) that code for putative integrases, and MGI-II contains a gene (PAM18_2243) that encodes a putative transposase. MGI-I contains another two genes, mod (PAM18_0704) and res (PAM18_0705), which encode two subunits of the type III restriction-modification (RM) system. MGI-II contains another three genes, $h s d R$ (PAM18_2237), hsdM (PAM18_2238) and hsdS (PAM18_2239) that code for all the three subunits in the multifunctional type I RM system which quite differ from the two type I RM systems in the PA7 genome. These two restriction systems contained in a single M18 genome can be predicated to assist this strain in digesting foreign genetic material [36], which may explain why $P$. aeruginosa M18 has proven to be recalcitrant to the introduction of foreign DNA and to keep the strain isolated from others within rhizosphere niches.

The GC content of MGI-III (65.6\%) is similar to that of the average content in the M18 genome. MGI-III contains a plt synthetic gene cluster with its two flanking regulatory genes and transporter gene cluster (pltMRLABCDEFGZHIJKNO) [37] and shares 99\% nucleotide sequence identity with the LESGI-2 sequence in LESB58, suggesting that the two islands may originate from the same genetic source and remain in the two genomes. However, LESGI-2 contains a frameshift mutation in pltB of the plt gene cluster [14]. The plt synthetic gene cluster is inactivated in strain LESB58, which might the result of the unnecessary Plt production in the nosocomial niche. However, Plt can be produced in the plant-associated pseudomonads such as 
A

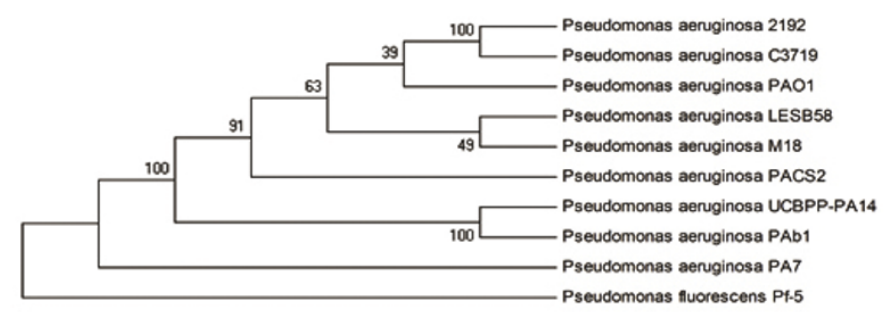

B
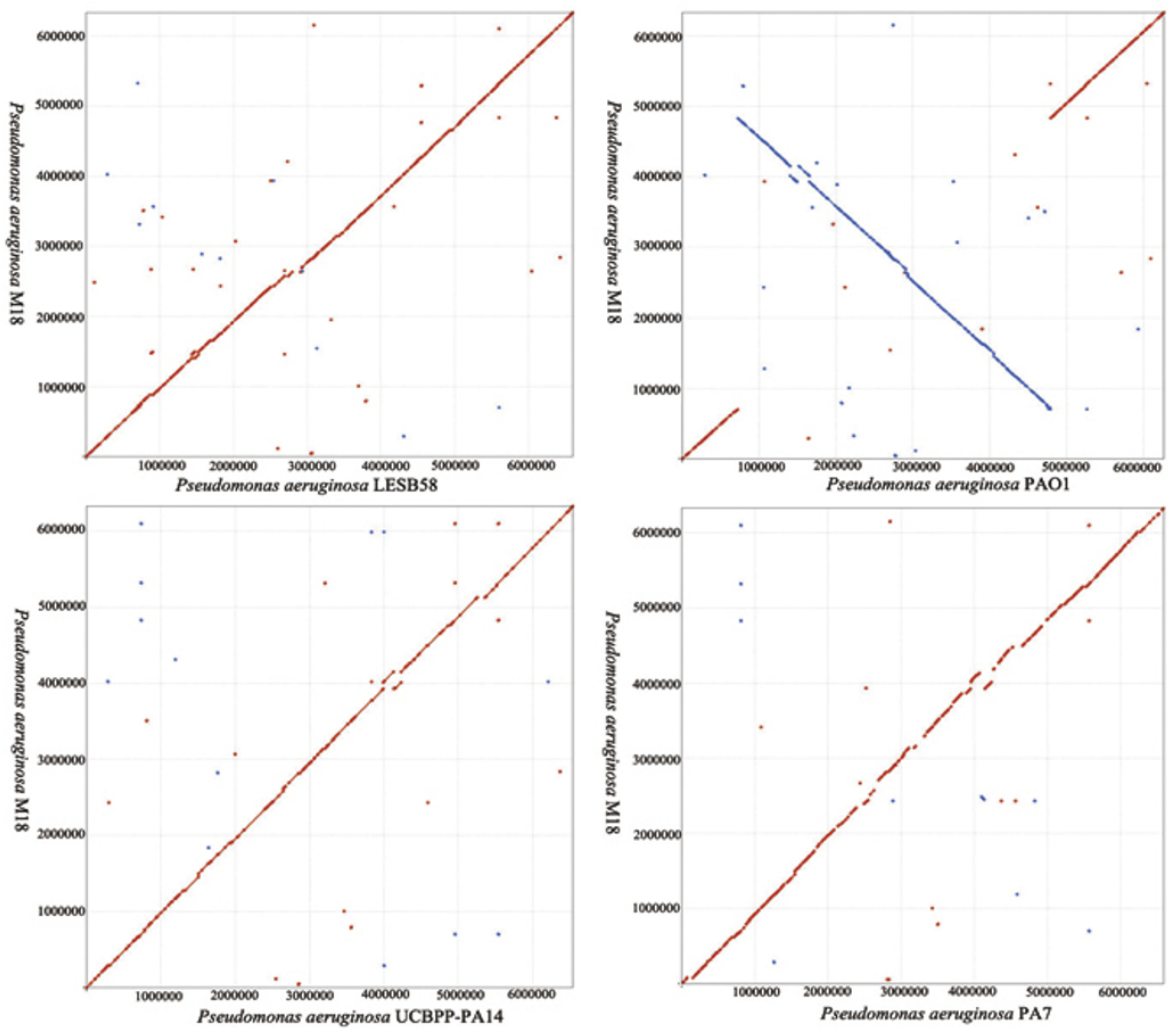

Figure 3 Genome alignment and phylogenetic analysis of $\boldsymbol{P}$. aeruginosa strains. A) Total 58 phylogenetically useful genes in Pseudomonas spp., which carries more than 10 parsimony informative sites, were selected to construct a phylogenetic tree [27]. The sequences of these strains were aligned by ClustalW [76], and a maximum parsimony (MP) phylogenetic tree was constructed by MEGA 4 [70] with bootstrap 1000. B) Genome alignment of $P$. aeruginosa M18 and other $P$. aeruginosa strains. Line figures depict the results of whole genome alignment results using MUMer [29]. The query genome sequence was the strain M18, and the subject genomes were the strains, LESB58, PAO1, PA14, and PA7. The red lines represent direct alignment, and the blue lines represent reverse alignment, respectively.

strain M18 [38] and P. fluorescens Pf-5 [39] and plays a crucial anti-fungal function to suppress a variety of plant diseases in rhizosphere niches.

The GC content of MGI-IV (63.1\%) and MGI-V (59.5\%) is closer to the average content of the M18 genome than those of MGI-I and MGI-II, indicating that the two islands may be not the recently acquired genetic materials. A homologous sequence of MGI-IV was found in P. aeruginosa PAK [40]. The MGI-IV contains genes encoding acetyltransferase, 3-demethylubiquinone-9,3-methyltransferase, CMP-2-keto-3- deoxyoctulosonic acid synthetase, phenylpropionate dioxygenase, ring-hydroxylating related dioxygenases, and nucleotide sugar transaminase, which are involved in flagellar 
A MGI- I

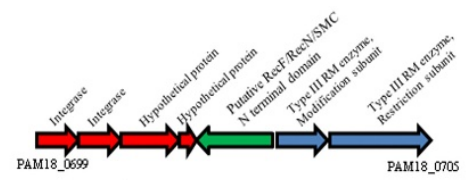

MGI-II

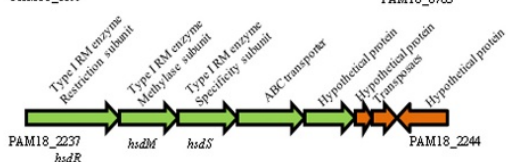

MGI-III

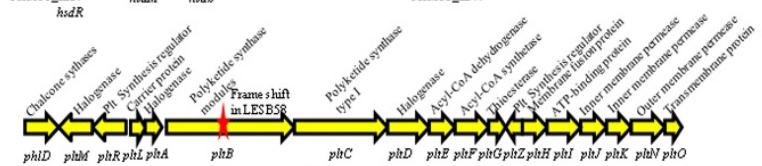

MGI-IV

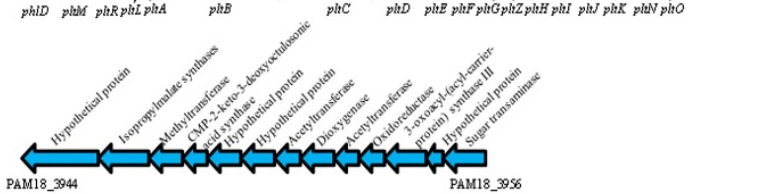

MGI.V

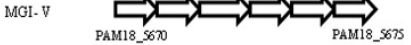

Prophage I

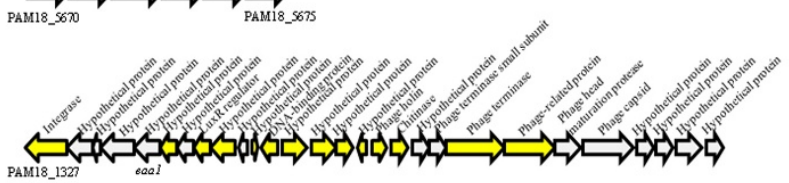

B

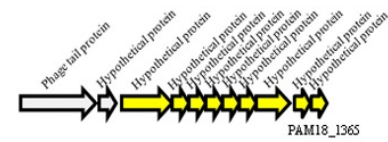

Prophage II
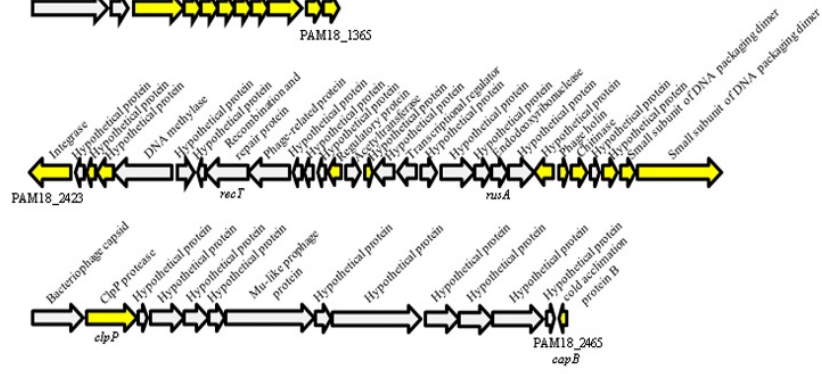

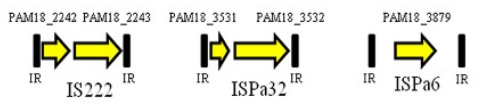

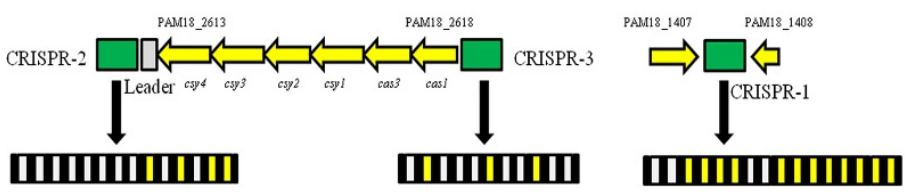

Figure 4 Genomic islands, prophages, IS and CRISPR loci in strain M18. A) Genomic islands (Gls) and prophages in the M18 genome. MGI-I contains mod and res genes coding for type III DNA restriction and modification system (RM) and the genes similar to those in Pseudomonas putida GB-1 (red), Pseudomonas syringae pv. Tabaci (ATCC11528) (deep green) and Stenotrophomonas sp. SKA14 (blue). MGI-II contains genes hsdR, hsdM and hsdS encoding for type I DNA RM and the genes similar to those in Nitrosomonas europaea (ATCC 19718) (green) and P. aeruginosa PA14 (orange). In MGI-III to V, genes similar to P. aeruginosa LESB58 (yellow); P. aeruginosa PAK (light blue) and the genes which had no significant similarity to other published genes in P. aeruginosa (white), The star symbol in MGI-III indicates the frameshift site in the pltB gene in LESB58. In prophages, yellow represents genes similar to $P$. aeruginosa LESB58 (share over 30\% identity); white represents genes that have no significant similarity to $P$. aeruginosa LESB58. B) The chromosomal organization of insertion sequences (ISS) and clustered, regularly interspaced short palindromic repeats (CRISPRs) with CRISPR-associated (cas) genes. In the IS loci, black boxes represent invert repeat (IR) sequences; IS coding genes are located between the two black boxes. The M18 genomic region between CRISPR-2 and CRISPR-3 covered the ORFs from PAM18_2613-PAM18_2618 and name of each are designated. Black boxes indicate the repeat sequence; gray boxes indicate the spacer sequences with over $70 \%$ identical sequences to other $P$. aeruginosa genome sequences; white boxes represent the spacers with dissimilar sequences to other $P$. aeruginosa strains. 
glycosylation, secondary metabolite synthesis, and redox reaction in the rhizosphere environment. The MGI-V is a novel genomic island which has never been found in other prokaryotic genomes. The gene (PAM18_5672) in this island encodes a putative cytochrome P450, which can catalyze the oxidation of several metabolic intermediates, such as lipids and steroidal hormones, as well as xenobiotic substances, including drugs and other toxic chemicals [41]. The two genomic islands in the M18 genome may assist the strain M18 to utilize various substances and degrade environmental toxic materials and pollutants in the complex rhizosphere environment.

Two novel prophages named prophage I and II were found in the M18 genome. Each of the prophage contains a putative chitinase gene (PAM18_1344 and PAM18_2447 [42]. Chitinase is a specific degradation enzyme that breaks down glycoside bonds in chitin and is essential for the degradation of chitin, a major component of the fungal cell wall. Besides the three copies of $c a p B$ gene in the M18 genome, an additional copy of $c a p B$ gene was identified in prophage II. The $c a p B$ gene is responsible for encoding a cold shock protein B involving in the adaptation to cold stress in the environment [43]. The two chitinase genes and an additional capB gene in the two prophages in the M18 genome suggest that the strain M18 has developed its anti-fungal and cold stress resistant genomic features under the strainspecific environmental selective pressures imposed on it.

\section{Insertion sequence (IS) and clustered regularly interspaced short palindromic repeats (CRISPR)}

A striking feature in the M18 genome is the least number and diversity of 3 IS elements named IS222 (1,227 bp), ISPa32 (1,232 bp) and ISPa6 (1,316 bp) as shown in Figure 4B). However, the other completely sequenced $P$. aeruginosa strains, PAO1, PA14, PA7, and LESB58 carry $12,10,15$ and 8 IS elements, respectively (Table 1). To our knowledge, the IS elements are postulated to be important drivers of many bacterial genome evolution, such as Xanthomonas [44]. In addition to serving as vectors for lateral gene transfer, IS elements can generate other types of genome modification, including rearrangements, inversions and deletions, any of which can increase the instability of the bacterial genome. Therefore, the least number of IS elements in the genome indicates the relative genome stability of the strain M18 in the rhizosphere niches.

As predicted by the CRISPRFinder [45], three CRISPR elements were found in the M18 genome (Figure 4B). CRISPR 1 element is $987 \mathrm{bp}$ in length and has 16 spacers each flanked by a 28 bp direct repeat (DR). There is one single similar CRISPR element in the LESB58 genome, in which six spacers are different from the CRISPR 1 element. The CRISPR 2 element consists of 866 bp with 14 spacers each flanked by 28 bp DR, and the CRISPR3 is $687 \mathrm{bp}$ in length with 11 spacers and 28 bp DR. The CRISPR 2 and 3 sequences are similar to those in the PA14 genome, which only has the two homologies. Furthermore, most of spacers in the CRISPR 2 and 3 elements are quite different from those in other $P$. aeruginosa strains. The strains PAO1 and PA7 only carry some questionable CRISPR elements that have borderline sequence identity. The three CRISPR elements in the M18 genome can be found dispersedly in other sequenced $P$. aeruginosa genomes, indicating that the M18 genome contains the most number of CRISPR elements among the five available complete $P$. aeruginosa genomes. The different spacer sequences in the three CRISPR loci of the strain M18 indicate that M18 has developed specific features to combat various phage invasions in the rhizosphere environment, as spacer sequences are known to be critical for bacteria to resist foreign phage invasion [46,47].

\section{Biocontrol-related gene clusters with their products}

We identified six secondary metabolite biosynthetic gene clusters, which are related to biocontrol activities of the strain M18, and responsible for biosynthesis of two siderophores, hydrogen cyanide, Plt and PCA (Additional file 3). Among them, two gene clusters are responsible for the biosynthesis of two siderophores, including pyoverdine (Pvd) and pyochelin (Pch), respectively, which have been demonstrated to suppress target phytopathogens in the rhizosphere through iron competition [48]. The hcn cluster responsible for the production of hydrogen cyanide $(\mathrm{HCN})$ is highly conserved in different $P$. aeruginosa strains. The Plt biosynthesis gene cluster is located in MGI-III and has been described above. The remaining two gene clusters are responsible for PCA biosynthesis. The two phz gene clusters in the genome of the strain M18 are highly conserved and share 99\% homologous sequences to those in the $P$. aeruginosa PAO1, each of which consists of seven genes (phzA1B1C1D1E1F1G1 and phzA2B2C2D2E2F2G2), named phzA1-G1 and phzA2-G2, respectively [49]. The genes that flank these two clusters are also the same to those of $P$. aeruginosa PAO1. However, there is a 520 bp intergenic region located directly downstream of the phzA2-G2 gene cluster between the phzG2 gene and the ORF (PAM18_3137) in the strain M18, compared to a shorter 96 bp sequence in the strain PAO1 and a relatively longer 286 bp sequence in the LESB58 genome. All these interval regions are consisted of clustered short repeats, although their function remains unknown.

The predominant phenazine produced in strain M18 is PCA, especially at $28^{\circ} \mathrm{C}$, rather than PYO produced in other nosocomial originating $P$. aeruginosa strains [11]. 
Both PCA and Plt compounds produced by fluorescent Pseudomonas species are considered to play an important role in microbial competitiveness and combating plant pathogens [50]. To our knowledge, the strain M18 is the first strain that was reported to produce both PCA and Plt simultaneously, and the synergistic antibiotic effect of PCA and Plt can result in strong activity to protect plants from fungal phytopathogen infections. It has been found that the wild-type strain M18 has much greater effective inhibitory activity against various mycelium plug growths than either the single PCA or Plt null mutants on the PDA plate (data not shown). We purified the two compounds of PCA and Plt individually from liquid culture, and measured the antifungal activities of the single compound and the combinations of various weight ratios of the two compounds against $R$ hizoctonia solani mycelium plug growth on PDA plates, respectively. The $50 \%$ value of efficient control $\left(\mathrm{EC}_{50}\right)$ is 8.38 and $58.13 \mu \mathrm{g} / \mathrm{ml}$ for single PCA and Plt, respectively. However, the $\mathrm{EC}_{50}$ of the two compound combination at a weight ratio of 10 to 1 at $\mathrm{pH} 7.0$ drops to $0.74 \mu \mathrm{g} / \mathrm{ml}$ only, indicating a significant synergistic effect of PCA and Plt combination on fungal inhibition. The combinations of the two antibiotics may play a crucial role for the strain M18 to protect the plant against different phytopathogen infections.

\section{Virulence-related genes against mammals}

Besides the virulence related genes located in the accessory genome, the LESB58 genome contains all of the
257 genes described for PAO1 with the exception of PA2399 ( $p v d D)$ and PA1392 [14]. Based on the VFDB database [51], we found that 14 out of 255 virulence genes in the M18 genome are quite different from those of LESB58 after BLASTP searching (Table 2). Firstly, six genes in the M18 genome (i.e., flgL, fliC, fla G, fliD, fliS and $f(i T)$ share less than $70 \%$ identity with the genes in LESB58, which are involved in the synthesis of flagellum components and related to bacterial motility and biofilm formation. Furthermore, the flagellum of the strain LESB58 belongs to a member of b-type because it lacks flagellar glycosylation, and the flagellum of the strain M18 may belong to the a-type resulted by the flagellar glycosylation function of MGI-IV in the M18 genome. These differences may give rise to different ability of bacterial biofilm formation and induce different mammalian immune system between the two strains. Secondly, five out of 42 pyoverdine synthesis-associated genes, which are involved in the iron uptake and cytotoxic due to its ability to stimulate the production of reactive oxygen species [52], are quite different between the two strains. The gene $p v d S$ (PALES28681) is completely absent in the M18 genome. Notably, there are three copies of $f p v A$ (encoding the first ferric pyoverdine receptor) and $p v d E$ (encoding an $\mathrm{ABC}$ transporter) in the LESB58 genome [14], but only one copy of $f p v A$ (PAM18_2643) and $p v d E$ (PAM18_2644) in the M18 genome; the two genes have only $24.9 \%$ and $57.7 \%$ sequence identity, respectively, to those in LESB58. Furthermore, $p v d D$ (PAM18_2640) and $p v d J$

Table 2 Divergence of virulence genes between P.aeruginosa LESB58 and M18.

\begin{tabular}{cccccc}
\hline Divergent virulence gene in LESB58 $^{\mathbf{a}}$ & Gene symbol & Length (AA) & Similar gene in M18 & Identity (\%) & Match length/Query length \\
\hline PALES_42341 & flgL & 439 & PAM18_3957 & 67.58 & 1 \\
PALES_42291 & fliC & 488 & PAM18_3943 & 48.26 & 1 \\
PALES_42281 & flaG & 123 & PAM18_3942 & 60 & 0.61 \\
PALES_42271 & fliD & 474 & PAM18_3941 & 42.76 & 0.98 \\
PALES_42261 & flis & 126 & PAM18_3939 & 66.39 & 0.97 \\
PALES_42251 & fliT & 98 & PAM18_3938 & 46.94 & 1 \\
PALES_49071 & pilA & 154 & PAM18_4617 & 71.61 & 1 \\
PALES_29051 & pvdE & 550 & PAM18_2644 & 57.71 & 1 \\
PALES_29021 & pvdE & 550 & PAM18_2644 & 57.71 & 1 \\
PALES_28991 & pvdE & 550 & PAM18_2644 & 57.71 & 1 \\
PALES_28971 & pvdD & 4,096 & PAM18_2640 & 57.21 & 1 \\
PALES_28961 & pvdJ & 1,123 & PAM18_2641 & 53.40 & - \\
PALES_28681 & pvdS & 155 & & -6 & 1 \\
PALES_28981 & fpvA & 819 & PAM18_0769 & 32.27 & - \\
PALES_29011 & fpvA & 819 & PAM18_0769 & 32.27 & 0.99 \\
PALES_29041 & fpvA & 819 & PAM18_2643 & 24.91 & 0.99 \\
PALES_00751 & ppkA & 1,032 & PAM18_0076 & 98.84 & 0.97 \\
PALES_27241 & lecA & 122 & PAM18_2466 & 100 & 0.58 \\
\hline
\end{tabular}

${ }^{\text {a }}$ Virulence genes in $P$. aeruginosa LESB58 were identified based on the VFDB database [51]. Divergent genes were identified by a BLASTP search under the expected level of $75 \%$ for identity or 0.7 for match length/query length.

b "_" indicates no homologous was found in the M18 genome. 
(PAM18_2641), which are predicted to encode the nonribosomal peptide synthetase in the pyoverdine synthesis pathway, have only $57.2 \%$ and $53.4 \%$ sequence identity to those in LESB58, respectively.

In the LESB58 genome, the $p p k A$ gene (PALES-00751) codes a positive regulator of type VI secretion system [53], and the gene lecA (PALES-27241) codes a factor which plays an important role in biofilm formation and adherence to mammalian cells [54]. However, both of the two homologous ppkA and lecA OFRs (PAM18_2466 and PAM18_0076) in M18 genome are truncated because of a stop codon located in the middle of the two ORFs.

The M18 genome carries a gene cluster responsible for lipopolysaccharide (LPS) O-antigen serotype O6 biosynthesis [32], which shares larger than $90 \%$ identity with that in LESB58. Interestingly, the gmd gene within the LPS biosynthesis gene cluster coding for GDP-mannose 4,6-dehydratase is a pseudogene in the LESB58 genome [14]. However, the gmd gene (PAM18_5576) was found to be a true gene in the M18 genome, although the association with the virulence and $\mathrm{O}$-antigen deficiency remains unclear.

\section{Attenuated competitive activity of the strain M18 in a mouse model of acute lung infection}

The different genome structures, especially the different mammalian virulence related genes, prompted us to make a comparison of the infectious activities between the two closely related strains M18 and LESB58 in a mammal model in vivo. Thus, we measured the competitive index $(\mathrm{CI})$ of the paired strains to assess their different ability in growth and maintenance in an acute mouse lung infection model (Figure 5). Equal ratio of the two strains was adjusted in phosphate buffered saline (PBS), and the mixture was inoculated into the mouse lung via the nostril. Bacteria were enumerated in the lung tissue at $24 \mathrm{~h}$ and $48 \mathrm{~h}$ post-infection. The geometric mean CI values were 0.3909 and 0.1695 , respectively. The results showed that the strain M18 was easier to be erased than the strain LESB58 in all of the 10 tested mice, and the growth and maintenance abilities of the M18 strain were more attenuated after a prolonged duration of inoculation than LESB58. The preliminary results indicated that the strain LESB58 is more difficult to be erased than the strain M18 in the acute mouse lung infection mode. Further experiments are now being carried out in this laboratory to elucidate the virulence activities of the strain M18 against humans and animal cell lines, and to identify the relationship between the virulence gene expression profiles of the M18 genome in the mouse lung and the responses of mammalian immune system.

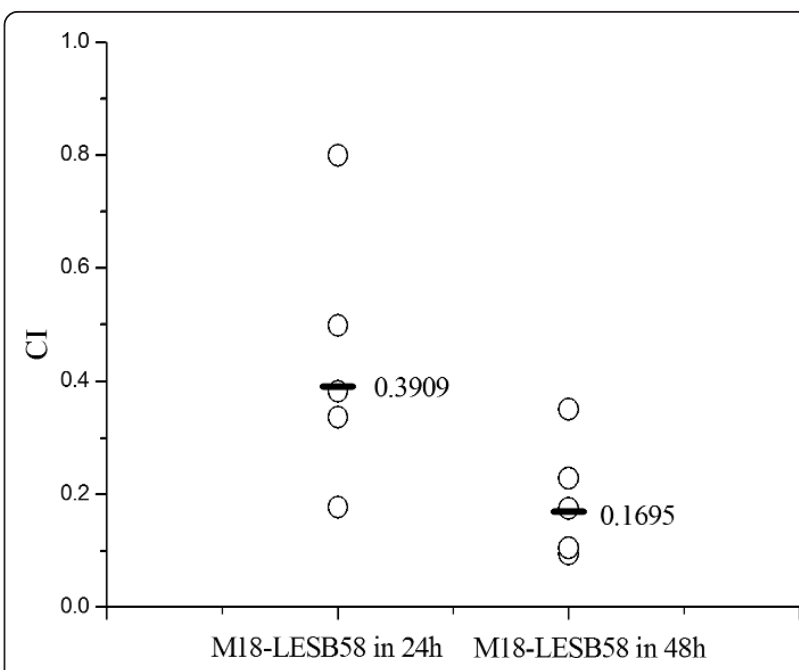

Figure 5 Competitive index analysis of strains M18 and LESB58 in a mouse model of acute lung infection. The competitive index (Cl) analysis of two strains, M18 and LESB58 at $24 \mathrm{~h}$ and $48 \mathrm{~h}$ after infection. The $\mathrm{Cl}$ is defined as the CFU output ratio of the strain M18 in comparison to the strain LESB58 divided by the CFU input ratio of the mixed suspension of both strains. Each circle represents the $\mathrm{Cl}$ for a single animal in each group. The $\mathrm{Cl}$ of less than 1 indicates a defect in growth and maintaince of the strain M18 in vivo than the strain LESB58. The geometric mean of the Cls for all mice is shown as a solid line.

\section{Antimicrobial agent resistance spectrum}

The minimal inhibitory concentration (MICs) of antimicrobial agents against the two strains M18 and LESB58 were listed in Table 3 . The strain M18 is phenotypically resistant to several antimicrobial agents including penicillins, cephalosporins, chloramphenicol, aminoglycosides and macrolides, which is the same as the reported $P$. aeruginosa strains [55]. The genomic analysis indicated that the strain M18 carries a $\beta$-lactamase gene ampC (PAM18_0829) and other eight putative $\beta$-lactamase genes (i.e., PAM18_0058, PAM18_1371, PAM18_2047, PAM18_2725, PAM18_3247, PAM18_4437, PAM18_5635, and PAM18_5664), which are responsible for encoding enzymes resistant to $\beta$-lactam antibiotics [56,57]. Furthermore, the M18 genome contains several gene clusters encoding proteins to constitute efflux pumps, such as mexAB-oprM (PAM18_0425-PAM18_0427) [58], mexCD-oprJ (PAM18_4693-PAM18_4691) [59], and mexEF-oprN (PAM18_2546-PAM18_2544) [60], which result in resistance to many antimicrobial agents including chloramphenicol, aminoglycoside, macrolides and rifampicin. Except the aztreonam, the strain M18 is susceptible to most of the clinically applied antimicrobial agents against $P$. aeruginosa, including third-generation cephalosporins (ceftazidime, ceftriaxone), carbapenems (imipenem, meropenem), monobactam (aztreonam), 
Table 3 Minimal inhibitory concentrations (MIC) of selected antimicrobial agents for P.aeruginosa M18 and LESB58.

\begin{tabular}{|c|c|c|c|}
\hline \multirow{2}{*}{$\begin{array}{l}\text { Class } \\
\text { Penicillins }\end{array}$} & \multirow{2}{*}{$\begin{array}{l}\text { Antimicrobial agents } \\
\text { Ampicillin }\end{array}$} & \multicolumn{2}{|c|}{$\begin{array}{l}\text { MIC }(\mu \mathrm{g} / \mathrm{mL}) \\
\text { M18 LESB58 }\end{array}$} \\
\hline & & 512 & 512 \\
\hline & Carbenicillin & 256 & 256 \\
\hline \multirow[t]{5}{*}{ Cephalosporins } & Cefotaxime & 256 & 256 \\
\hline & Cefminox & 256 & 256 \\
\hline & Cefoxitin & 256 & 256 \\
\hline & Ceftazidime & 2 & 2 \\
\hline & Ceftriaxone & 8 & 8 \\
\hline \multirow[t]{2}{*}{ Carbapebems } & Imipenem & 1 & 2 \\
\hline & Meropenem & 2 & 4 \\
\hline Monobactam & Aztreonam & 64 & 64 \\
\hline \multirow[t]{4}{*}{ Aminoglycoside } & Kanamycin & 16 & 512 \\
\hline & Gentamycin & 2 & 8 \\
\hline & Tobramycin & 0.5 & 0.5 \\
\hline & Spectinomycin & 256 & 256 \\
\hline Polymyxins & Colistin & 8 & 8 \\
\hline Tetracyclines & Tetracycline & 16 & 32 \\
\hline Macrolides & Roxithromycin & 32 & 32 \\
\hline Chloramphenicol & Chloramphenicol & 64 & 64 \\
\hline Rifampicines & Rifampicin & 128 & 128 \\
\hline Quinolones & Ofloxacin & 1 & 4 \\
\hline
\end{tabular}

polymyxin (colistin) and tobramycin. However, comparing with the strain LESB58, the strain M18 is slightly more susceptible to imipenem and meropenem.

Interestingly, compared with $P$. aeruginosa LESB58, the strain M18 is more susceptible to several antimicrobial agents, such as gentamycin, kanamycin and ofloxacin. Quinolone resistance is usually present in $P$. aeruginosa strains because of two point mutations in gyrA and parC in the genome, respectively [61]. The lack of the two point mutations in the M18 genome can be postulated to responsible for quinolone susceptibility of the strain M18. Therefore, the results suggest that the rhizosphere isolate M18 has not evolved specific mechanisms including point mutation or acquisition resistant plasmid to resist antimicrobial agents, unlike nosocomial $P$. aeruginosa strains that face lethal antimicrobial agents stress.

\section{Genomic microarray based transcriptome analysis}

Measurement of gene expression levels at two temperatures, $28^{\circ} \mathrm{C}$ and $37^{\circ} \mathrm{C}$, was performed using an oligonucleotide Agilent microarray based on the M18 genome sequence to specify the temperature-dependent expression profiles of this strain. We selected $28^{\circ} \mathrm{C}$ as a temperature representative for the rhizosphere niches and $37^{\circ} \mathrm{C}$ for the human body. The results from the temperature-dependent transcriptome analysis were consistent with our previously published data that the expression of phzM, ptsP and lasI gene is up-regulated at $37^{\circ} \mathrm{C}$ [11]. The comparative analysis of the M18 genome expressional profiles at $28^{\circ} \mathrm{C}$ and $37^{\circ} \mathrm{C}$ indicated that the expression levels of a total of 605 genes at $28^{\circ} \mathrm{C}$ are regulated over two-fold that at $37^{\circ} \mathrm{C}$. It shows that a total of $10.6 \%$ of the expressed genes in M18 genome are temperature-dependent (Additional file 4).

In comparison with gene expression level at $37^{\circ} \mathrm{C}$, the transcriptional expression levels of 277 genes are upregulated by approximately two-fold at $28^{\circ} \mathrm{C}$, including 151 genes with known functions and 126 functions unknown genes. Among these, 77 function known genes are sorted into nine gene families (Table 4). Surprisingly, 24 of these genes are contained in the M18 GIs and prophage, of which eight genes are located in prophage I, two genes are involved in the type I restriction-modification system in MGI-II, seven genes are related to the pyoluteorin transporter in MGI-III, and seven genes in MGI-V are associated with the synthesis of cytochrome P450. We also found that other up-regulated operons are involved in energy production and the conversion of TCA cycle-related oxidative phosphorylation, aromatic amino acid and benzonate degradation, and phosphate uptake. Besides the operons, a potential phenazine modifying enzyme, $p h z H$, a gene that encoded quorum sensing (QS) signal molecule $N$-butanoyl-L-homoserine lactone synthetase, rhlI, and a gene that encoded the QS repressor, $q s c R$, are promoted at $28^{\circ} \mathrm{C}$. The other genes, which are not located in these operons, could be assigned to various functions, including phage-related, FeS cluster assembly, glyoxylate metabolism, benzonate degradation, copper resistance, and pentose phosphate. Hence, the expression levels of these genes involved in antibiotics production, foreign genetic material prevention and nutrient substance degradation are up-regulated at the rhizosphere temperature $28^{\circ} \mathrm{C}$, which may give M18 an advantage to resistance the rhizosphere environmental stresses.

In comparison with the gene expression levels at $37^{\circ} \mathrm{C}$, a total of 328 gene expression levels are down-regulated by approximately two-fold at $28^{\circ} \mathrm{C} ; 53$ of these genes have unknown functions, and 189 function known genes are sorted into 19 gene families (Table 4), such as the secretion system, transportation, and ribosomal assembly and etc. These results indicated that the basic protein synthesis, metabolism rate and cell growth rate decrease at the low temperature of $28^{\circ} \mathrm{C}$ compared to $37^{\circ} \mathrm{C}$. Strikingly, none of them is located in M18 GIs or prophages. Interestingly, we found that the 16 downregulated genes are predicted to encode several mammalian virulence-related factors, including three genes predicated to encode $\mathrm{HCN}$ synthetase, three encode proteins involved in type II secretion system, eight genes 
Table 4 Transcriptomic analysis of P.aeruginosa $\mathrm{M} 18$ at $28^{\circ} \mathrm{C}$ and $37^{\circ} \mathrm{C}$

\begin{tabular}{|c|c|c|}
\hline $\begin{array}{l}\text { Gene family or gene } \\
\text { location }\end{array}$ & Gene ID or name & $\begin{array}{l}\text { Fold } \\
\text { change }\end{array}$ \\
\hline \multicolumn{3}{|l|}{ Up-regulated gene at $28^{\circ} \mathrm{C}$} \\
\hline Prophage I & PAM18_1347, PAM18_1349-1350, PAM18_1352-1355 & $1.8-3.4$ \\
\hline MGI ॥ & $h s d R, h s d M$ & $1.8-2.4$ \\
\hline MGI III & pltR, pltZ, pltHIJNO & $1.4-2.7$ \\
\hline MGI V & PAM18_5670-PAM18_5675 & $2.8-5.4$ \\
\hline Phage related & PAM18_0614, PAM18_0616-617, PAM18_0619, PAM18_0620, PAM18_0622-0625, PAM18_0626, PAM18_0627 & 2.3-8.4 \\
\hline TCA cycle & PAM18_1548-PAM1550 & $2.1-2.2$ \\
\hline Oxidative phosphorylation & cyoC, cyoA, nape, napF, napD, napABC & $1.5-2.7$ \\
\hline Fes cluster assembly & iscS, iscU, iscA & $1.8-2.2$ \\
\hline Glyoxylate metabolism & $f d n l, f d n H, f d n G$ & $1.6-1.6$ \\
\hline Benzonate degradation & amiE, PAM18_1600, amiC, amiR, PAM18_1603, bkdB, bkdA1, bkdA2 & $1.7-4.5$ \\
\hline Phosphate uptake & PAM18_1584, phnE, PAM18_1586, phnJ, phoQ, phoP & $1.6-2.7$ \\
\hline Copper resistance & $p C O A, p c O B$ & $1.4-1.5$ \\
\hline Pentose phosphate & $r b s k, r b s R, r b s A, r b s B$ & $1.6-1.9$ \\
\hline Regulatory proteins & rhll, ascR, PAM18_0792, PAM18_0975, PAM18_3016, PAM18_0807, PAM18_3383 & $2.0-6.1$ \\
\hline \multicolumn{3}{|l|}{$\begin{array}{l}\text { Down-regulated genes at } \\
28^{\circ} \mathrm{C}\end{array}$} \\
\hline Type III secretion system & exoT, exsD, PAM18_0335, exsC, popD, popB, pcrH, pcrV & $2.1-2.8$ \\
\hline Type II secretion system & $x c p P, x c p R, x c p T, \sec Y$ & $1.6-2.7$ \\
\hline $\begin{array}{l}\text { Protease production and } \\
\text { secretion }\end{array}$ & lasA, aprA, aprD, aprE, aprF, pfpl, PAM18_0458 & $2.3-11.4$ \\
\hline HCN synthesis & $h \subset n A, h \subset n B, h \subset n C$ & $1.5-2.9$ \\
\hline $\begin{array}{l}\text { Phenazine metabolism and } \\
\text { transport }\end{array}$ & phzA1-G1, phzM, phzA2-G2, opmD, mexl, mexH & $1.6-11.6$ \\
\hline Fimbrial biogenesis & cupC1, pilA, pilN & $2.2-3.0$ \\
\hline AHL biosynthesis & lasl & 2.5 \\
\hline Phospholipase C & plcB & 3.3 \\
\hline Detoxification & PAM18_1269, PAM18_1457, PAM18_1515, PAM18_4192 & $2.2-4.6$ \\
\hline Benzonate degradation & PAM18_0222-0224, PAM18_0250, phhABC, phhR, aroP2, hpd, hmgA, antABC, catABC & $2.1-57.0$ \\
\hline Transportation & $\begin{array}{l}\text { PAM18_0605-0606, PAM18_1782-1783, PAM18_1785, PAM18_2831-2832, PAM18_3039, PAM18_3041-3043, } \\
\text { PAM18_5348-5350, PAM18_4688, PAM18_1003 }\end{array}$ & $2.0-13.3$ \\
\hline Sulfur metabolism & PAM18_3206-3207, PAM18_2369-2372 & $2.1-3.4$ \\
\hline Iron uptake & PAM18_4792-4793, PAM18_4818-4819, PAM18_3537, PAM18_3606, PAM18_4448, PAM18_4605 & \\
\hline Amino acid metabolism & liuA, liuD, liuR, PAM18_3044-3046, arcABC & \\
\hline Heat shock proteins & ibpA, dnak, groEL & $2.1-3.5$ \\
\hline $\begin{array}{l}\text { Energy production and } \\
\text { conversion }\end{array}$ & narC, narE, nuoEGHIJLMN, atpABCDFGH, nirM, nirS, suCABCD, IpdG, PAM18_3490-3491 & 1.6-4.1 \\
\hline Ribosomal proteins & rp/ABCDEFJKLMNOPQRSWX, rpmCDEHJ, rpsCEFGHJKLMNQRS & $1.5-3.8$ \\
\hline Regulatory proteins & $\begin{array}{l}\text { PAM18_0249, PAM18_0710, PAM18_0945, PAM18_0966, PAM18_1156, PAM18_1507, PAM18_2031, } \\
\text { PAM18_2078, PAM18_2114, PAM18_2280, PAM18_2571, PAM18_2608, PAM18_3035, PAM18_3129, } \\
\text { PAM18_3270, PAM18_3440, PAM18_4328, PAM18_5219, PAM18_5273 }\end{array}$ & 2.0-3.9 \\
\hline
\end{tabular}

Cells were grown to $\mathrm{OD}_{600}=5.0-6.0$ (late exponential phase) in $\mathrm{LB}$ medium at $28^{\circ} \mathrm{C}$ and $37^{\circ} \mathrm{C}$, respectively. The effects on gene expression were monitored by microarray analysis using agilent GeneChips. Shown are genes that were located in Genomic islands or are member of gene families in two independent experiments.

involved in the type III secretion system, and two genes involved in LasA protease precursor and phospholipase $C$, respectively.

Transcriptome profile analysis based on the M18 genomic microarray at the two different temperatures implies that the M18 genome has developed its specific features for gene expression to meet the requirement for the competitive fitness and survival in rhizosphere niches except the specific genome structure features.

\section{Conclusions}

The strain M18 isolated from the sweet melon rhizosphere in 1996 in Shanghai suburb was named Pseudomonas sp. M18 because of its effective biocontrol ability 
and several phenotypic features that are similar to Pseudomonas spp. $[1,2,11]$. In this study, the complete genome of the strain M18 was sequenced and shown to share a common core genome that is comprised of more than $90 \%$ genome sequence homology to all other sequenced genomes of $P$. aeruginosa isolates (except taxonomic outlier PA7). Based on the comparative genomic and phylogenetic analysis of the M18 genome with other $P$. aeruginosa, the strain M18 can now be definitively designated as a new $P$. aeruginosa strain. The results indicated that the core genome diversity of various $P$. aeruginosa strains from either nosocomial or rhizosphere niche is quite small in comparison with that of various $P$. fluorescens strains [62].

However, the various $P$. aeruginosa strains originating from different environment niches differ in accessory genome regions, genome expression profiles, virulence activities and antibiotic resistances. The analysis of complete M18 genome sequence and temperature dependent transcriptional profiles indicated several important features distinguished the strain M18 from other nosocomial $P$. aeruginosa strains. Firstly, the M18 genome contains several specific accessory regions of genomic plasticity which differ significantly from all sequenced nosocomial isolates. This is the first time to sequence completely a rhizosphere originating $P$. aeruginosa strain M18, and take it as a model strain to analysis and detail the possible determinants related to its biocontrol activity and living ability in rhizosphere niche. We found that the M18 genome contains five specific GIs and two novel prophages, which benefit its survival and biocontrol activity in the rhizosphere niches. Furthermore, all mammalian pathogenicity-related GIs and prophages contained in other sequenced $P$. aeruginosa strains [14,33-35] are absent in M18 genome. Secondly, fourteen mammalian virulence-related genes are absent or truncated in M18 genome, and the competitive index analysis of the strains M18 and LESB58 indicated the strain M18 is easier to be erased than that of LESB58 in the acute infection mouse lung model. Comparing with the strain LESB58, the strain M18 is more susceptible to several antibiotics and antimicrobial agents including kanamycin, gentamycin and ofloxacin. Thirdly, the M18 genome based microarray profiles revealed that the strain M18 has developed its specific temperaturedependent gene expression patterns to meet the requirements of survival and thriving in the rhizosphere niche. Especially, we found that none in the GIs expressed higher at $37^{\circ} \mathrm{C}$ than that at $28^{\circ} \mathrm{C}$, but 24 genes in the GIs and prophages are up-regulated at $28^{\circ} \mathrm{C}$ which is the temperature close to that in rhizosphere niches.

In summary, the rhizosphere originated strain M18 has evolved specific genome structures and the temperature dependent expressional patterns to meet the requirement of fitness and survival under the selective pressure imposed on it. Therefore, the specific interesting features found in M18 genome and transcriptome profiles may provide us several cues to design therapeutic strategies against $P$. aeruginosa infection, and assist us to develop the strain M18 or other rhizosphere originating strains as a safety cell factory for the industrial purposes to produce antifungal compounds or other secondary metabolites through genetically engineered modification.

\section{Methods}

\section{Bacterial strains, medium, and growth conditions}

$P$. aeruginosa M18 was isolated from sweet melon rhizosphere in Shanghai suburb in 1996 and maintained in our laboratory. P. aeruginosa LESB58 which is a hypervirulent strain isolated from CF patient [63], was obtained from Dr. Robert E.W. Hancock. The two strains were typically grown in Luria Bertani (LB) medium at $28^{\circ} \mathrm{C}$ and $37^{\circ} \mathrm{C}$, respectively.

\section{Genome sequencing}

The draft $P$. aeruginosa M18 genome sequence was obtained using GS 20 system (454 Life Science Corporation). Overall, 547,645 high-quality reads were assembled and had an average length that covered 32.9fold of the genome, and yielded 227 contigs with the 454 assembly tool. Among these, 172 large contigs (> $500 \mathrm{bp}$ ) represented $99.18 \%$ of the draft sequence. The complete sequence was assembled and finished according to the method described by Zhang [64], and the average error rate of the finished genome was lower than $1 \mathrm{bp}$ in $10 \mathrm{~kb}$.

\section{Nucleotide sequence accession number}

The annotated genome sequence has been deposited into GenBank under accession no. СР002496.

\section{Genome annotation}

Genome annotation of the strain M18 was mainly performed as described previously [64]. In brief, putative coding sequences (CDS) were predicted using Glimmer 3 [65], and the short CDS (<90 bp) were eliminated. The putative protein function were predicted using BLASTP searches against all the annotated proteins of the $P$. aeruginosa strains, LESB58, PAO1, UCBPP-PA14 and PA7, and the $\mathrm{nr}$ (non-redundant protein sequences) database in NCBI based on the most significant matches. Clusters of orthologous groups (COGs) and subcellular localizations for each CDS were predicted using RPS-BLAST [66], PSORTb v.2.0 [67] and LipoP1.0 [68]. Transfer RNAs were predicted by TRNASCAN-SE 
[69]. Ribosomal RNAs and small RNAs were predicted by BLASTN searches against those of $P$. aeruginosa strains, LESB58, PAO1, UCBPP-PA14 and PA7. ISs were identified by the IS Finder database http://www-is. biotoul.fr/, and CRISPRs were predicted by CRISPRFinder [45].

\section{Comparative genomics}

The $P$. aeruginosa M18 genome sequence was aligned to those of other sequenced $P$. aeruginosa genomes currently at NCBI with the use of MUMmer [29]. The M18 strain-specific CDS were identified with mGenomeSubtractor [28] with $75 \%$ identities, or $70 \%$ length ratios of matching regions to questionable CDSs.

\section{Phylogenetic analysis}

Nine completely sequenced $P$. aeruginosa genomes were selected to assess the phylogenetic status of M18, while P. fluorescens Pf-5 was used as a member of the "out" group. A total of 58 phylogenetically useful genes [27] which carried more than 10 parsimony informative sites, were selected to construct the phylogenetic tree. Then, the nucleotide sequences of 58 PUG genes in these strains were connected, aligned, and constructed to form a maximum parsimony tree using MEGA 4 software [70].

\section{Genomic island identification}

GIs and prophages located in the 3'-end of the annotated tRNA genes were firstly identified using MobilomeFINDER [71]. GIs located in the other sites of M18 genome were identified by mGenomeSubtractor [28]. The large islands that contained more than five CDS were kept. The direct repeat (DR) elements were identified using BLASTN searches. Prophages were identified by manual inspection of phage-related genes.

\section{Fungal growth inhibition assay}

Fungal growth inhibition by $P$. aeruginosa strain M18 was determined by measuring its ability to inhibit the growth of a plug of Mycosphaerella melonis mycelium on a potato dextrose agar plate (PDA). Briefly, a plug in 5 - $\mathrm{mm}$ diameter from the leading edge of $M$. melonis culture grown for 5 days at $28^{\circ} \mathrm{C}$ on a PDA plate was taken and placed in the center of a fresh PDA plate. The strain M18 cultures grown overnight on a King's medium B (KMB) plate were placed at the position 25 $\mathrm{mm}$ away from the mycelium plug on PDA plate. The co-culture plates were incubated at $28^{\circ} \mathrm{C}$ and scored after 4 or 5 days by measuring the distance between the edges of the bacterial colony and the fungal mycelium plug [72]. Each experiment was repeated twice with three replicates.

\section{Competitive index analysis in mouse}

The acute lung infection mouse model has been previously described [73]. Five mice were used for each experiment. According to the guidelines of ethics committee for animal treatment, adult female BALB/c mice that weighted more than $17 \mathrm{~g}$ at the age of 6 - to 8weeks-old were used. The animals were anesthetized using $2.5 \%$ avertin by intraperitoneal administration. The bacteria were centrifuged and washed twice with PBS, and the final concentration was adjusted to approximately $1 \times 10^{8} \mathrm{CFU} / \mathrm{ml}$. Equal ratio of the culture of the two strains M18 and LESB58 was mixed for inoculation. With the mice held in an upright position, $10 \mu \mathrm{l}$ of the bacterial mixture was inoculated twice (20 $\mu \mathrm{l}$ in total) into mice via application into the nostril with a pipette. Mice were sacrificed at $24 \mathrm{~h}$ and $48 \mathrm{~h}$ post-inoculation and their lungs were used for CFU counts based on the significant colony size differences of the two strains on LB plates, as the colony diameter of M18 colony and LESB58 is 1-2 $\mathrm{mm}$ and $0.2-0.5 \mathrm{~mm}$ in size after overnight culture, respectively. The two different strain colonies were further verified by colony PCR using phzG2 interval region primer, and the PCR products of M18 and LESB58 colonies are 1075 bp and 841 bp in length, respectively. The pair of primer sequences is designed as phzG2Iup: ACTGACACTGAGGTGCGAAAGCG; phzG2Idown: ACCGTATGCGCTTCACTTGACC. The M18/LESB58 CIs in vivo were determined according to the method previously described [73].

\section{Minimum inhibitory concentration (MIC) Determination}

The MIC determination method was applied to measure the susceptibility of the two strains of $P$. aeruginosa M18 and LESB58 to a range of antimicrobial agents, as previously described [25]. For each antimicrobial agent, a serial of two-fold diluted agent was generated using an appropriate starting concentration in Müller-hinton broth media. The MIC corresponds to the smallest agent concentration that inhibits the growth of $P$. aeruginosa M18 and LESB58.

\section{Oligonucleotide microarray experiments}

The $P$. aeruginosa M18-specific Agilent Oligonucleotide microarray (Gene Expression Omnibus (GEO) Platform: GPL11372) was designed with the eArray software. For each of 5,684 annotated CDS, a 60-mer oligonucleotide was designed and repeated twice on the microarray. The $P$. aeruginosa strain M18 was cultured overnight in LB medium with $180 \mathrm{rpm}$ at $28^{\circ} \mathrm{C}$ and $37^{\circ} \mathrm{C}$, respectively. The two cultures were inoculated by 100 -fold diluted into LB medium for three biological replicates and grown to $\mathrm{OD}_{600} \sim 5.0-6.0$. The total RNA was extracted 
using TRIzol (Invitrogen) and measured with a BioAnalyzer (Agilent Technologies, Palo Alto, CA, USA). Total RNA was eluted in RNase-free water and purified by QIAGEN RNeasy kit. A total of $2 \mu \mathrm{g}$ RNA was reversetranscribed to cDNA by a one-step method. The cDNA was transcribed into RNA by T7 RNA polymerase, modified by aa-UTP at $40^{\circ} \mathrm{C}$, before labeling with Cy3 fluorescence dye at $25^{\circ} \mathrm{C}$, purified by a QIAGEN RNeasy mini kit, and quantified by the BioAnalyzer. After hybridization, the arrays were washed according to the manufacturer instructions and scanned twice by an Agilent scanner at $100 \%$ and $10 \%$ photo multiplier tube (PMT). The normalized data was analyzed using an R script [74]. Expression levels of the genes were compared using SBC analysis system (http://sas.ebioservice.com/). Average values of each gene were calculated based on the three biological replicates.

\section{Oligonucleotide microarray data accession number} The microarray data has been deposited in to GEO under accession no. GSE26518.

\section{Additional material}

\section{Additional file 1: Strain specific genes of $P$. aeruginosa M18. The strain-specific genes were predicted by proteins with low similarity to the other four sequenced $P$. aeruginosa strain genomes (under the expected threshold of 0.6 for identity or 0.7 for match length/query length) using the mGenomeSubtractor [28].}

Additional file 2: General features of genomic islands and prophages in $P$. aeruginosa M18 genome. The general features of five genomic islands and two prophages were described, including position, ORF numbers, GC content, mobility gene, specific coding genes, homologous regions and predicted function.

Additional file 3: Secondary metabolite biosynthesis gene clusters in $P$. aeruginosa M18. The gene clusters for producing five secondary metabolites in strain M18 genome.

Additional file 4: Detailed comparative transcriptomic analysis of $P$. aeruginosa $\mathrm{M} 18$ at $28^{\circ} \mathrm{C}$ and $37^{\circ} \mathrm{C}$. the complete lists of genes differentially regulated over two-fold with a $p$-value of 0.05 or lower at the two temperatures of $28^{\circ} \mathrm{C}$ and $37^{\circ} \mathrm{C}$.

\section{Acknowledgements}

We are grateful to the sequencing team at Chinese National Human Genome Center at Shanghai for their assistance of sequencing and annotation; ShanghaiBio Corporation for assistance of microarray experiment and analysis. This work was funded by the National Key Basic Research Program (973 Program, No. 2009CB118906), the National High Technology Research and Development Program of China (863 Program, No. 2006AA10A209 and 2007AA02Z215), the Shanghai Science and Technology Program (No. 08391911900), and the Shanghai Leading Academic Discipline Project (No. B203)

\section{Authors' contributions}

YQX, DQW and HYO conceived and designed the experiments. DQW and YWH HYO were responsible for sequencing, finishing and annotating. DQW, YQX, YWH, JY and XW performed experiments and data analysis. YQX, HYO and $\mathrm{XQH}$ contributed reagents/materials/analysis tools. YQX, DQW, YWH and HYO wrote the paper. All authors read and approved the final manuscript.
Received: 29 July 2011 Accepted: 31 August 2011

Published: 31 August 2011

\section{References}

1. Hu H, Xu Y, Chen F, Zhang X, Hur BK: Isolation and characterization of a new fluorescent Pseudomonas strain that produces both phenazine 1carboxylic acid and pyoluteorin. J Microbiol Biotechnol 2005, 15:86-90.

2. Ge Y, Huang X, Wang S, Zhang X, Xu Y: Phenazine-1-carboxylic acid is negatively regulated and pyoluteorin positively regulated by gac $A$ in Pseudomonas sp. M18. FEMS Microbiol Lett 2004, 237:41-47.

3. Ge $Y$, Pei D, Feng P, Huang $X, X u Y$ : Autoinduction of RpoS biosynthesis in the biocontrol strain Pseudomonas sp. M18. Curr Microbiol 2007, 54:97-101

4. Wang $Y$, Huang $X, H u H$, Zhang $X, X u Y$ : QscR acts as an intermediate in gacA-dependent regulation of PCA biosynthesis in Pseudomonas sp. M18. Curr Microbiol 2008, 56:339-345.

5. Yan A, Huang X, Liu H, Dong D, Zhang D, Zhang X, Xu Y: An rhl-like quorum-sensing system negatively regulates pyoluteorin production in Pseudomonas sp. M18. Microbiology 2007, 153:16-28.

6. Chen $Y$, Wang $X$, Huang $X$, Zhang $X, X u$ Y: Las-like quorum-sensing system negatively regulates both pyoluteorin and phenazine-1carboxylic acid production in Pseudomonas sp. M18. Sci China C Life Sci 2008, 51:174-181.

7. Zhang X, Wang S, Geng H, Ge Y, Huang X, Hu H, Xu Y: Differential regulation of rsmA gene on biosynthesis of pyoluteorin and phenazine1-carboxylic acid in Pseudomonas sp. M18. World J Microbiol Biotechnol 2005, 21:883-889.

8. Gurusiddaiah S, Weller DM, Sarkar A, Cook RJ: Characterization of an antibiotic produced by a strain of Pseudomonas fluorescens inhibitory to Gaeumannomyces graminis var. tritici and Pythium spp. Antimicrob Agents Chemother 1986, 29:488-495.

9. Howell CR, Stipanovic RD: Suppression of Pythium ultimum-induced damping-off of cotton seedlings by Pseudomonas fluorescens and its antibiotic, pyoluteorin. Phytopathology 1980, 70:712-715.

10. Chin-A-Woeng TFC, Bloemberg GV, van der Bij AJ, van der Drift $K$, Schripsema J, Kroon B, Scheffer RJ, Keel C, Bakker P, Tichy HV, et al: Biocontrol by phenazine-1-carboxamide-producing Pseudomonas chlororaphis PCL1391 of tomato root rot caused by Fusarium oxysporum f. sp. radicis-lycopersici. Molecular Plant-Microbe Interactions 1998, 11:1069-1077.

11. Huang J, Xu Y, Zhang H, Li Y, Huang X, Ren B, Zhang X: Temperaturedependent expression of phzM and its regulatory genes lasl and pts $P$ in rhizosphere isolate Pseudomonas sp. strain M18. Appl Environ Microbiol 2009, 75:6568-6580.

12. Mavrodi DV, Blankenfeldt W, Thomashow LS: Phenazine compounds in fluorescent Pseudomonas spp. biosynthesis and regulation. Annu Rev Phytopathol 2006, 44:417-445.

13. Gibson J, Sood A, Hogan DA: Pseudomonas aeruginosa-Candida albicans interactions: localization and fungal toxicity of a phenazine derivative. Appl Environ Microbiol 2009, 75:504-513.

14. Winstanley C, Langille MG, Fothergill JL, Kukavica-Ibrulj I, Paradis-Bleau C, Sanschagrin F, Thomson NR, Winsor GL, Quail MA, Lennard N, et al: Newly introduced genomic prophage islands are critical determinants of in vivo competitiveness in the Liverpool Epidemic Strain of Pseudomonas aeruginosa. Genome Res 2009, 19:12-23.

15. Lu J, Huang $X$, Zhang M, Li S, Jiang H, Xu Y: The distinct quorum sensing hierarchy of las and $r h /$ in Pseudomonas sp. M18. Curr Microbiol 2009, 59:621-627.

16. Buysens S, Heungens K, Poppe J, Hofte M: Involvement of pyochelin and pyoverdin in suppression of Pythium-Induced damping-off of tomato by Pseudomonas aeruginosa 7NSK2. Appl Environ Microbiol 1996, 62:865-871.

17. Anjaiah $V$, Cornelis $P$, Koedam N: Effect of genotype and root colonization in biological control of fusarium wilts in pigeonpea and chickpea by Pseudomonas aeruginosa PNA1. Can J Microbiol 2003, 49:85-91.

18. Bano N, Musarrat J: Characterization of a new Pseudomonas aeruginosa strain NJ-15 as a potential biocontrol agent. Curr Microbiol 2003, 46:324-328.

19. Kumar RS, Ayyadurai N, Pandiaraja P, Reddy AV, Venkateswarlu Y, Prakash O, Sakthivel N: Characterization of antifungal metabolite produced by a new strain Pseudomonas aeruginosa PUPa3 that exhibits broad-spectrum 
antifungal activity and biofertilizing traits. J Appl Microbiol 2005, 98:145-154.

20. Li Y, Jiang H, Du $X$, Huang $X$, Zhang $X, X u Y$ : Enhancement of phenazine1-carboxylic acid production using batch and fed-batch culture of gacA inactivated Pseudomonas sp. M18G. Bioresour Technol 2010, 101:3649-3656.

21. Su J, Zhou Q, Zhang H, Li Y, Huang X, Xu Y: Medium optimization for phenazine-1-carboxylic acid production by a gacA qscR double mutant of Pseudomonas sp. M18 using response surface methodology. Bioresour Technol 2010, 101:4089-4095.

22. Zhou $Q$, Su J, Jiang $H$, Huang $X$, Xu Y: Optimization of phenazine-1carboxylic acid production by a gacA/qscR-inactivated Pseudomonas sp. M18GQ harboring pME6032Phz using response surface methodology. Appl Microbiol Biotechnol 2010, 86:1761-1773.

23. Stover CK, Pham XQ, Erwin AL, Mizoguchi SD, Warrener P, Hickey MJ, Brinkman FS, Hufnagle WO, Kowalik DJ, Lagrou M, et al: Complete genome sequence of Pseudomonas aeruginosa PAO1, an opportunistic pathogen. Nature 2000, 406:959-964.

24. Lee DG, Urbach JM, Wu G, Liberati NT, Feinbaum RL, Miyata S, Diggins LT, He J, Saucier M, Deziel E, et al: Genomic analysis reveals that Pseudomonas aeruginosa virulence is combinatorial. Genome Biol 2006, 7: R90.

25. Roy PH, Tetu SG, Larouche A, Elbourne L, Tremblay S, Ren Q, Dodson R, Harkins D, Shay R, Watkins $K$, et al: Complete genome sequence of the multiresistant taxonomic outlier Pseudomonas aeruginosa PA7. PLOS ONE 2010, 5:e8842.

26. Wolfgang MC, Kulasekara BR, Liang X, Boyd D, Wu K, Yang Q, Miyada CG, Lory S: Conservation of genome content and virulence determinants among clinical and environmental isolates of Pseudomonas aeruginosa. Proc Natl Acad Sci USA 2003, 100:8484-8489.

27. Mathee K, Narasimhan G, Valdes C, Qiu X, Matewish JM, Koehrsen M, Rokas A, Yandava CN, Engels R, Zeng E, et al: Dynamics of Pseudomonas aeruginosa genome evolution. Proc Natl Acad Sci USA 2008, 105:3100-3105.

28. Shao Y, He X, Harrison EM, Tai C, Ou HY, Rajakumar K, Deng Z: mGenomeSubtractor: a web-based tool for parallel in silico subtractive hybridization analysis of multiple bacterial genomes. Nucleic Acids Res 2010, 38(Suppl):W194-200

29. Delcher AL, Phillippy A, Carlton J, Salzberg SL: Fast algorithms for largescale genome alignment and comparison. Nucleic Acids Res 2002, 30:2478-2483.

30. Klockgether J, Munder A, Neugebauer J, Davenport CF, Stanke F, Larbig KD, Heeb S, Schock U, Pohl TM, Wiehlmann L, Tummler B: Genome diversity of Pseudomonas aeruginosa PAO1 laboratory strains. J Bacteriol 2010, 192:1113-1121.

31. Winsor GL, Van Rossum T, Lo R, Khaira B, Whiteside MD, Hancock RE, Brinkman FS: Pseudomonas Genome Database: facilitating user-friendly, comprehensive comparisons of microbial genomes. Nucleic Acids Res 2009, 37:D483-488.

32. Raymond CK, Sims EH, Kas A, Spencer DH, Kutyavin TV, Ivey RG, Zhou Y, Kaul R, Clendenning JB, Olson MV: Genetic variation at the O-antigen biosynthetic locus in Pseudomonas aeruginosa. J Bacteriol 2002, 184:3614-3622.

33. He J, Baldini RL, Deziel E, Saucier M, Zhang Q, Liberati NT, Lee D, Urbach J, Goodman HM, Rahme LG: The broad host range pathogen Pseudomonas aeruginosa strain PA14 carries two pathogenicity islands harboring plant and animal virulence genes. Proc Natl Acad Sci USA 2004, 101:2530-2535.

34. Harrison EM, Carter ME, Luck S, Ou HY, He X, Deng Z, O'Callaghan C, Kadioglu A, Rajakumar K: Pathogenicity islands PAPI-1 and PAPI-2 contribute individually and synergistically to the virulence of Pseudomonas aeruginosa strain PA14. Infect Immun 2010, 78:1437-1446.

35. Battle SE, Meyer F, Rello J, Kung VL, Hauser AR: Hybrid pathogenicity island PAGI-5 contributes to the highly virulent phenotype of a Pseudomonas aeruginosa isolate in mammals. J Bacteriol 2008, 190:7130-7140.

36. Kobayashi I: Behavior of restriction-modification systems as selfish mobile elements and their impact on genome evolution. Nucleic Acids Res 2001, 29:3742-3756.

37. Huang $X$, Yan A, Zhang $X, X u Y$ : Identification and characterization of a putative $A B C$ transporter PItHIJKN required for pyoluteorin production in Pseudomonas sp. M18. Gene 2006, 376:68-78.
38. Huang $X$, Zhu D, Ge Y, Hu H, Zhang $X, X u$ Y: Identification and characterization of pltZ, a gene involved in the repression of pyoluteorin biosynthesis in Pseudomonas sp. M18. FEMS Microbiol Lett 2004, 232:197-202.

39. Nowak-Thompson B, Chaney N, Wing JS, Gould SJ, Loper JE: Characterization of the pyoluteorin biosynthetic gene cluster of Pseudomonas fluorescens Pf-5. J Bacteriol 1999, 181:2166-2174.

40. Arora SK, Bangera M, Lory S, Ramphal R: A genomic island in Pseudomonas aeruginosa carries the determinants of flagellin glycosylation. Proc Natl Acad Sci USA 2001, 98:9342-9347.

41. Werck-Reichhart D, Feyereisen R: Cytochromes P450: a success story. Genome Biol 2000, 1:REVIEWS3003.

42. Bhattacharya D, Nagpure A, Gupta RK: Bacterial chitinases: properties and potential. Crit Rev Biotechnol 2007, 27:21-28.

43. Schindelin H, Jiang W, Inouye M, Heinemann U: Crystal structure of CspA, the major cold shock protein of Escherichia coli. Proc Natl Acad Sci USA 1994, 91:5119-5123.

44. Monteiro-Vitorello CB, de Oliveira MC, Zerillo MM, Varani AM, Civerolo E, Van Sluys MA: Xylella and Xanthomonas Mobil'omics. OMICS 2005, 9:146-159.

45. Grissa I, Vergnaud G, Pourcel C: CRISPRFinder: a web tool to identify clustered regularly interspaced short palindromic repeats. Nucleic Acids Res 2007, 35:W52-57.

46. Brouns SJ, Jore MM, Lundgren M, Westra ER, Slijkhuis RJ, Snijders AP, Dickman MJ, Makarova KS, Koonin EV, van der Oost J: Small CRISPR RNAs guide antiviral defense in prokaryotes. Science 2008, 321:960-964.

47. Marraffini LA, Sontheimer EJ: CRISPR interference limits horizontal gene transfer in staphylococci by targeting DNA. Science 2008, 322:1843-1845.

48. Briat JF: Iron assimilation and storage in prokaryotes. J Gen Microbiol 1992, 138:2475-2483.

49. Li Y, Du X, Lu ZJ, Wu D, Zhao Y, Ren B, Huang J, Huang X, Xu Y: Regulatory feedback loop of two phz gene clusters through $5^{\prime}$-untranslated regions in Pseudomonas sp. M18. PLOS ONE 2011, 6:e19413.

50. Miller SH, Mark GL, Franks A, O'Gara F: Pseudomonas-Plant Interactions WileyVCH Verlag GmbH \& Co. KGaA; 2008.

51. Yang J, Chen L, Sun L, Yu J, Jin Q: VFDB 2008 release: an enhanced webbased resource for comparative pathogenomics. Nucleic Acids Res 2008, 36:D539-542.

52. Meyer JM, Neely A, Stintzi A, Georges C, Holder IA: Pyoverdin is essential for virulence of Pseudomonas aeruginosa. Infect Immun 1996, 64:518-523.

53. Mougous JD, Gifford CA, Ramsdell TL, Mekalanos JJ: Threonine phosphorylation post-translationally regulates protein secretion in Pseudomonas aeruginosa. Nat Cell Biol 2007, 9:797-803.

54. Diggle SP, Stacey RE, Dodd C, Camara M, Williams P, Winzer K: The galactophilic lectin, LecA, contributes to biofilm development in Pseudomonas aeruginosa. Environ Microbiol 2006, 8:1095-1104.

55. Zavascki AP, Carvalhaes CG, Picao RC, Gales AC: Multidrug-resistant Pseudomonas aeruginosa and Acinetobacter baumannii: resistance mechanisms and implications for therapy. Expert Rev Anti Infect Ther 2010, 8:71-93.

56. Jacoby GA: AmpC beta-lactamases. Clin Microbiol Rev 2009, 22:161-182, Table of Contents.

57. Livermore DM, Woodford N: The beta-lactamase threat in Enterobacteriaceae, Pseudomonas and Acinetobacter. Trends Microbiol 2006, 14:413-420.

58. Li XZ, Nikaido H, Poole K: Role of mexA-mexB-oprM in antibiotic efflux in Pseudomonas aeruginosa. Antimicrob Agents Chemother 1995, 39:1948-1953.

59. Poole K, Gotoh N, Tsujimoto H, Zhao Q, Wada A, Yamasaki T, Neshat S, Yamagishi J, Li XZ, Nishino T: Overexpression of the mexC-mexD-oprJ efflux operon in $n f x B$-type multidrug-resistant strains of Pseudomonas aeruginosa. Mol Microbiol 1996, 21:713-724.

60. Kohler T, Michea-Hamzehpour M, Henze U, Gotoh N, Curty LK, Pechere JC: Characterization of MexE-MexF-OprN, a positively regulated multidrug efflux system of Pseudomonas aeruginosa. Mol Microbiol 1997, 23:345-354.

61. Jalal S, Wretlind B: Mechanisms of quinolone resistance in clinical strains of Pseudomonas aeruginosa. Microb Drug Resist 1998, 4:257-261.

62. Silby MW, Cerdeno-Tarraga AM, Vernikos GS, Giddens SR, Jackson RW, Preston GM, Zhang XX, Moon CD, Gehrig SM, Godfrey SA, et al: Genomic and genetic analyses of diversity and plant interactions of Pseudomonas fluorescens. Genome Biol 2009, 10:R51. 
63. Cheng K, Smyth RL, Govan JR, Doherty C, Winstanley C, Denning N, Heaf DP, van Saene H, Hart CA: Spread of beta-lactam-resistant Pseudomonas aeruginosa in a cystic fibrosis clinic. Lancet 1996, 348:639-642.

64. Zhang ZY, Liu C, Zhu YZ, Zhong Y, Zhu YQ, Zheng HJ, Zhao GP, Wang SY, Guo XK: Complete genome sequence of Lactobacillus plantarum JDM1. J Bacteriol 2009, 191:5020-5021.

65. Delcher AL, Harmon D, Kasif S, White O, Salzberg SL: Improved microbial gene identification with GLIMMER. Nucleic Acids Res 1999, 27:4636-4641.

66. Marchler-Bauer A, Panchenko AR, Shoemaker BA, Thiessen PA, Geer LY, Bryant SH: CDD: a database of conserved domain alignments with links to domain three-dimensional structure. Nucleic Acids Res 2002, 30:281-283.

67. Gardy JL, Laird MR, Chen F, Rey S, Walsh CJ, Ester M, Brinkman FS: PSORTb v.2.0: expanded prediction of bacterial protein subcellular localization and insights gained from comparative proteome analysis. Bioinformatics 2005, 21:617-623.

68. Juncker AS, Willenbrock $H$, Von Heijne G, Brunak S, Nielsen H, Krogh A: Prediction of lipoprotein signal peptides in Gram-negative bacteria. Protein Sci 2003, 12:1652-1662.

69. Lowe TM, Eddy SR: tRNAscan-SE: a program for improved detection of transfer RNA genes in genomic sequence. Nucleic Acids Res 1997, 25:955-964.

70. Tamura K, Dudley J, Nei M, Kumar S: MEGA4: Molecular Evolutionary Genetics Analysis (MEGA) software version 4.0. Mol Biol Evol 2007, 24:1596-1599.

71. Ou HY, He X, Harrison EM, Kulasekara BR, Thani AB, Kadioglu A, Lory S, Hinton JC, Barer MR, Deng Z, Rajakumar K: MobilomeFINDER: web-based tools for in silico and experimental discovery of bacterial genomic islands. Nucleic Acids Res 2007, 35:W97-W104.

72. Thomashow LS, Weller DM: Role of a phenazine antibiotic from Pseudomonas fluorescens in biological control of Gaeumannomyces graminis var. tritici. J Bacteriol 1988, 170:3499-3508.

73. Kukavica-lbrulj I, Bragonzi A, Paroni M, Winstanley C, Sanschagrin F, OToole GA, Levesque RC: In vivo growth of Pseudomonas aeruginosa strains PAO1 and PA14 and the hypervirulent strain LESB58 in a rat model of chronic lung infection. J Bacteriol 2008, 190:2804-2813.

74. Ihaka R, Gentleman R: R: a language for data analysis and graphics. J Comput Graph Stat 1996, 5:299-314.

75. Ghai R, Hain T, Chakraborty T: GenomeViz: visualizing microbial genomes. BMC Bioinformatics 2004, 5:198.

76. Larkin MA, Blackshields G, Brown NP, Chenna R, McGettigan PA, McWilliam H, Valentin F, Wallace IM, Wilm A, Lopez R, et al: Clustal W and Clustal $\times$ version 2.0. Bioinformatics 2007, 23:2947-2948.

doi:10.1186/1471-2164-12-438

Cite this article as: Wu et al:: Genomic analysis and temperature-

dependent transcriptome profiles of the rhizosphere originating strain Pseudomonas aeruginosa M18. BMC Genomics 2011 12:438.

\section{Submit your next manuscript to BioMed Central and take full advantage of:}

- Convenient online submission

- Thorough peer review

- No space constraints or color figure charges

- Immediate publication on acceptance

- Inclusion in PubMed, CAS, Scopus and Google Scholar

- Research which is freely available for redistribution

Submit your manuscript at www.biomedcentral.com/submit
Biomed Central 